
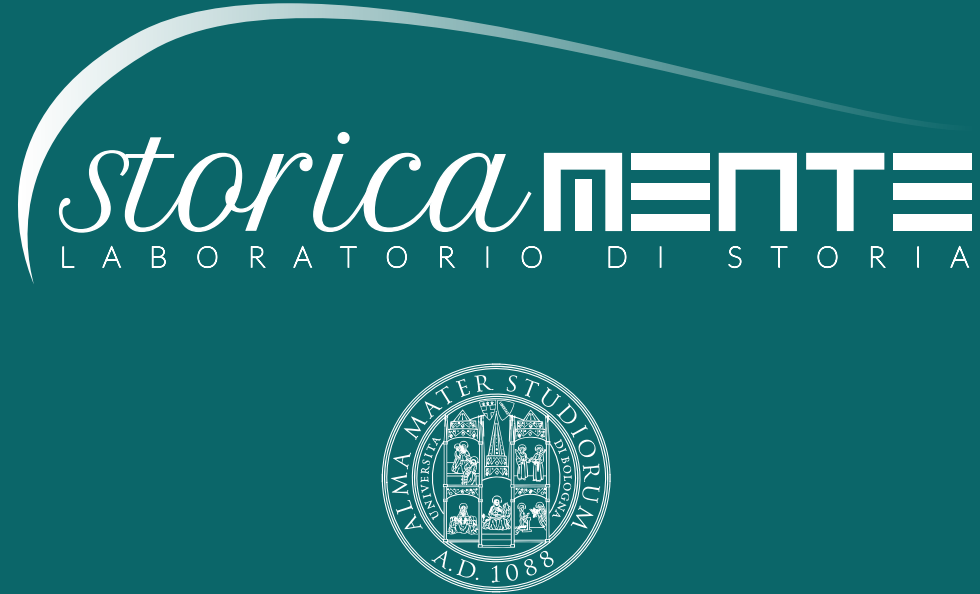

ALMA MATER STUDIORUM

Università di Bologna

Dipartimento di Storia Culture Civiltà

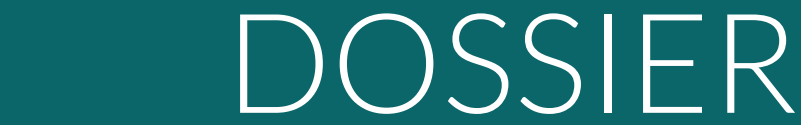

IMPERIALISMI E RETAGGI POSTCOLONIALI IN ITALIA, PORTOGALLO, SPAGNA 


\section{STORICAMENTE.ORG}

\section{Laboratorio di Storia}

Fernando Tavares Pimenta

La decolonizzazione e l'indipendenza del Mozambico: coloni bianchi e movimenti nazionalisti

Numero 12 - 2016

ISSN: 1825-411X

Art. 26

pp. 1-38

DOI: $10.12977 /$ stor646

Editore: BraDypUS

Data di pubblicazione: 30/12/2016

Sezione: Dossier "Imperialismi e retaggi postcoloniali in Italia, Portogallo, Spagna” 


\title{
La decolonizzazione e l'indipendenza del Mozambico: coloni bianchi e movimenti nazionalisti
}

\author{
FERNANDO TAVARES PIMENTA \\ Univ. Nova de Lisboa, \\ Instituto Português de Relações Internacionais
}

The essay analyzes the historical process of independence of Mozambique, especially the political behavior of the white population during the phase of decolonization (1974/1975). We will analyze the different forms of decolonization and the different positions of various political factions and groups within the white community, as well as the positions taken by the Portuguese State, as a colonial power, and by Frelimo, as the main nationalist movement. Our goal is to understand the evolution of the independence process from the inside of colonial society and, at the same time, understand the reasons of the rebellion of September 7, 1974, of the exclusion of the white population from politics and of its flight abroad in August 1974.

Traduzione dal portoghese di Tiziana Zenier In questo saggio andremo ad analizzare il processo storico dell'indipendenza del Mozambico, tenendo in particolare considerazione il comportamento politico della popolazione bianca durante la fase della decolonizzazione (1974/1975). Innanzitutto, però, è necessario delineare il contesto storico mozambicano nel periodo coloniale.

Posto sul confine tra l'Africa Orientale e l'Africa Australe, bagnato dalle acque dell'Oceano Indiano, il Mozambico consta di una superficie di 799.380 chilometri quadrati. Le sue frontiere furono stabilite in base ad accordi internazionali stipulati durante il periodo coloniale tra portoghesi, inglesi, tedeschi e boeri, il che portò alla formazione di un territorio con una lunga linea di costa, ma con un ridotto entroterra, spe- 
cialmente al sud. Vari popoli africani, neri, con pochi o nessun legame tra loro, furono aggregati all'interno delle frontiere coloniali. Sono cinque i principali gruppi etno-linguistici mozambicani: Macua-Lomwe, Maconde e Yao, a nord del fiume Zambesi, e gli Shona e i Thonga a sud [Dias 1964]. Sulla costa c'è anche da rilevare una significativa influenza islamica e, in alcuni casi, indiana.

D'altro canto, i portoghesi si erano stabiliti in modo permanente in alcune città e in punti della costa e della valle del fiume Zambesi fin dal XVI secolo, specificatamente nell'Ilha de Moçambique, a Quelimane e a Tete. Tuttavia, la maggior parte del territorio fu occupato militarmente dalle forze portoghesi solo alla fine del XIX secolo, se non addirittura nei primi due decenni del XX secolo. Risale a quest'epoca lo sviluppo delle due città principali: Lourenço Marques (la capitale, l'attuale Maputo') e Beira, due città portuali e sedi di due importanti linee ferroviarie fondamentali per le comunicazioni con i territori vicini,

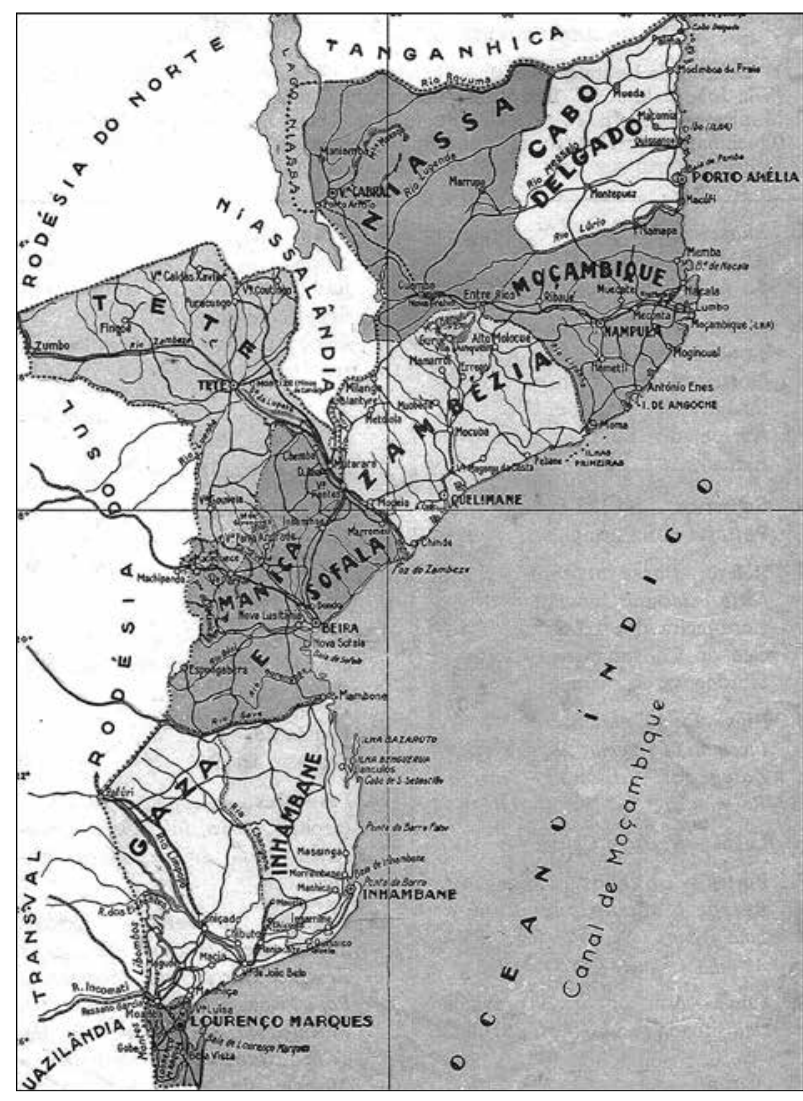

Mappa del Mozambico durante il periodo coloniale. Fonte: http://dx.doi.org/10.1590/S0104-59702014000200006

Nella stesura del saggio manterremo la toponomastica utilizzata nel periodo coloniale. 
in particolare con il Transvaal, nell'Africa del Sud, e con la Rhodesia del Sud (Zimbabwe) [Newitt 1995].

A parte queste città, il popolamento bianco fu abbastanza rilevante in alcune zone della valle del fiume Limpopo (nel sud) e nell'altipiano di Chimoio (Vila Pery, nel centro). Negli Anni ‘60, furono creati alcuni insediamenti bianchi nelle fertili zone dei distretti di Niassa e di Cabo Delgado (nel nord), mentre avanzava celere lo sviluppo della città di Nampula. Così, i coloni bianchi erano passati da 17.842 nel 1928, a 27.438 nel 1940, 48.213 nel 1950, 97.245 nel 1960 e 162.967 nel 1970. All'epoca costituivano circa il 2\% del totale della popolazione mozambicana, che si aggirava sugli 8.168 .933 abitanti². Da notare anche che nelle località di più antica colonizzazione c'era una significativa popolazione meticcia, che in larga misura si identificava con il Portogallo. In termini politici, il Mozambico faceva parte di un Impero coloniale estremamente centralizzato. In ultima analisi, il potere risiedeva nel governo di Lisbona, che nominava e dimetteva a suo piacimento il Governatore Generale, figura massima della gerarchia dello Stato coloniale. L'intervento dei coloni e della restante popolazione nel governo era molto ridotto. Il Consiglio Legislativo, creato nel 1955, aveva una funzione poco più che consultiva $\mathrm{e}$, ancorché le sue funzioni fossero state ampliate alla fine del governo di Marcelo Caetano, non ebbe mai un vero potere, né una vera autonomia rispetto al potere esecutivo. D'altra parte, lo Stato Portoghese impediva la formazione e lo sviluppo di partiti politici sia tra i coloni che tra la popolazione africana. I sindacati e la stampa erano strettamente controllati dal regime di António Oliveira Salazar, il cosiddetto Estado Novo ${ }^{3}$, specificatamente dalla censura e dalla

Nel 1970, oltre i 162.967 bianchi, risiedevano nella colonia circa 50.189 meticci, 22.531 indiani, 3.814 cinesi e 7.929 .432 neri. Sul popolamento bianco dei territori africani si veda Pimenta 2014 ed anche Castelo 2007.

Anche dopo il 1945 Salazar resistette alla democrazia e, più tardi, alla decolonizzazione dell'Impero Coloniale in Africa. Nel settembre 1968, Salazar fu esonerato, per motivi di salute, dalla carica di Presidente del Consiglio dei Ministri. Morì due anni 
polizia politica portoghese, la famigerata Polizia Internazionale di Difesa dello Stato (Polícia Internacional de Defesa do Estado, PIDE) [Hedges 1993; Isaacman 1983].

\section{I movimenti di opposizione: il Frelimo}

Durante i periodi di campagna elettorale per l'Assemblea Nazionale (di Lisbona) e per la Presidenza della Repubblica, la dittatura permise la comparsa di liste di opposizione. Fu in questo contesto che sorse il Movimento di Unità Democratica (Movimento de Unidade Democrática, MUD), nel 1945, più tardi severamente represso dal regime, o la candidatura di opposizione del Generale Humberto Delgado alla Presidenza della Repubblica, nel 1958. In Mozambico, l'opposizione democratica esercitò una consistente influenza politica, soprattutto a Lourenço Marques e a Beira. Questi oppositori erano per lo più avvocati ed altri liberi professionisti, quasi tutti bianchi, che non dipendevano direttamente dall'amministrazione pubblica e che disponevano di uno status sociale ben consolidato nella società coloniale, il che li rendeva tollerati dal regime [Santos 2006; 2016].

Dall'altra parte, l'Estado Novo rigettò categoricamente l'autonomia politica del Mozambico, rifiutandosi di discutere il problema della decolonizzazione anche quando le altre potenze europee cominciarono

più tardi, il 27 luglio 1970. Dopo alcune esitazioni, il Presidente della Repubblica, Américo Tomaz, invitò Marcelo Caetano, Professore di Giurisprudenza dell'Università di Lisbona, a formare il governo. Il 27 settembre 1968, Marcelo Caetano prese posto come Presidente del Consiglio dei Ministri, mettendo la parola fine a trentasei anni di governo diretto di Salazar. Marcelo Caetano delineò un tentativo fallito di liberalizzazione politica del regime e di modificazione della politica coloniale a favore della concessione di una maggiore autonomia amministrativa per le colonie africane. Ma questa autonomia avrebbe dovuto beneficiare in particolare i coloni bianchi residenti in Angola ed in Mozambico. Il fallimento del suo governo, soprattutto sul piano coloniale, determinò la caduta del regime tramite un golpe militare il 25 aprile 1974. Cfr. Cruz 1980, Rosas 1990, Pinto 1992, Torgal 2009, Pimenta 2011. 
a preparare l'indipendenza delle rispettive colonie nei decenni del 1950 e del 1960. E tanto meno accettò il formarsi di movimenti nazionalisti nelle colonie africane. Questa situazione obbligò i mozambicani con ideali nazionalisti ad organizzarsi politicamente in clandestinità $\mathrm{e}$, di fronte alle difficoltà di svolgere le proprie attività all'interno della colonia, ad andare in esilio. $\mathrm{Fu}$ in questo contesto che venne fondato il Fronte di Liberazione del Mozambico (Frente de Libertação de

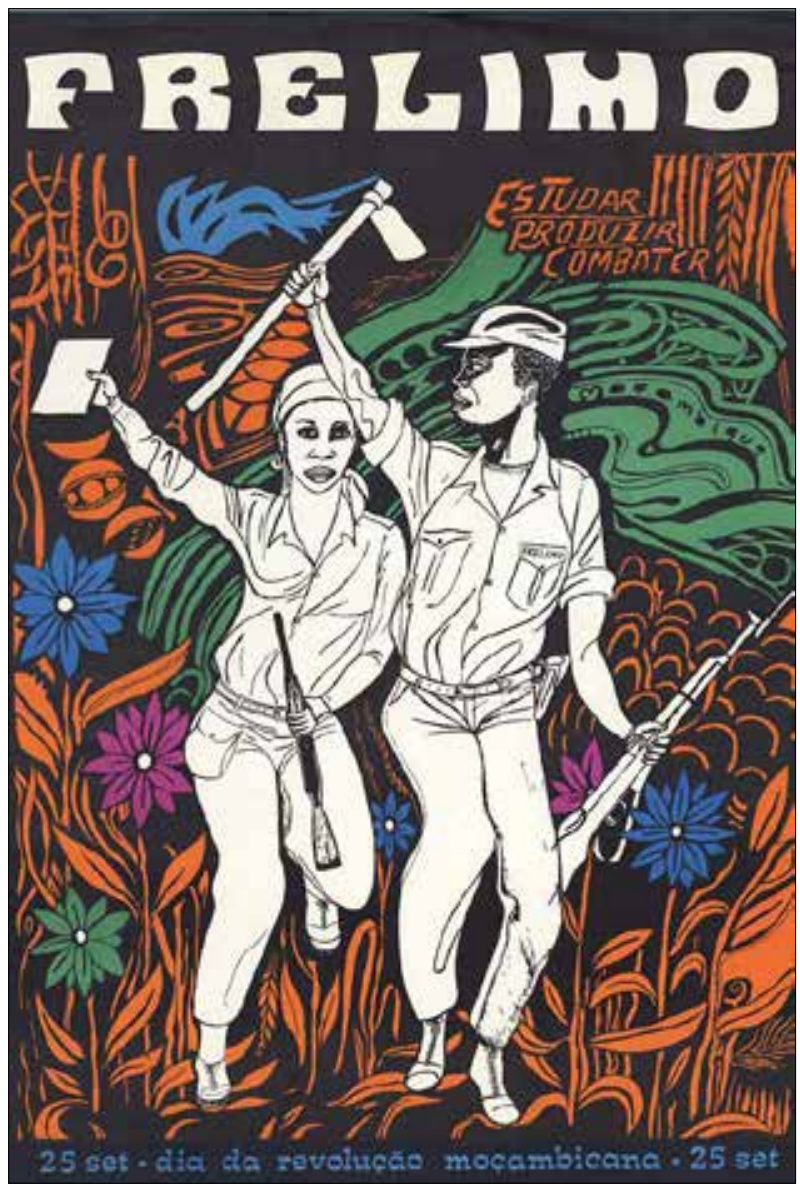

Frelimo, Manifesto sulla Commemorazione della rivoluzione in Mozambico. Grafica Agostinho Milhafre. Tanzania, 1973. Fonte: International Institute of Social History, call nr: BG D17/463 Moçambique, Frelimo), a Dar-es-Salaam, in Tanzania, il 25 giugno 1962. Il Frelimo nacque dalla fusione di tre organizzazioni nazionaliste preesistenti: l'União Democrática Nacional de Moçambique (UDENAMO), la Mozambique African National Union (MANU) e l'União Nacional Africana de Moçambique Independente (UNAMI). Eduardo Mondlane, un intellettuale nero dottore in sociologia e professore all'Università di Syracuse, negli Stati Uniti d'America, fu il primo Presidente del Frelimo [Mondlane 1969]. Da notare che i quadri del Frelimo erano per lo più neri, ma compren- 
devano anche un numero significativo di meticci, di indiani e finanche alcuni bianchi.

Il rifiuto portoghese a negoziare l'indipendenza della colonia portò il Frelimo ad abbracciare la lotta armata per l'indipendenza il 25 settembre del 1964. La guerriglia si scatenò nella provincia settentrionale di Cabo Delgado, vicino al confine con la Tanzania. Il Portogallo rispose col rafforzamento del dispositivo militare, facendosi coinvolgere in un terzo conflitto coloniale, dopo quello dell'Angola (1961) e quello della Guinea (1963). Più tardi, il Frelimo aprì un altro fronte di combattimento nella regione di Tete, vicino alla frontiera con lo Zambia, obbligando le forze portoghesi a disperdersi. Tuttavia, la guerra rimase distante dai centri urbani e dalle zone di colonizzazione bianca, il che trasmise ai coloni la falsa idea che la situazione fosse sotto controllo da parte dell'esercito [Afonso, Gomes 2010].

Nel frattempo, il 3 febbraio 1969, Eduardo Mondlane fu ucciso dall'esplosione di una bomba carta presumibilmente preparata dalla Polizia Internazionale di Difesa dello Stato. Dopo l'assassinio di Mondlane, il comando del movimento fu assunto dal Vice Presidente, il reverendo (nero) Uria Simango. Simango era considerato un elemento politicamente più conservatore e meno favorevole al proseguimento di una linea socialista rivoluzionaria dominante nel Comitato Centrale. Avrebbe avuto anche una visione più essenzialista del processo di emancipazione del popolo mozambicano, che identificava con la maggioranza nera. Per questo, guardava con una certa diffidenza alla partecipazione di meticci, indiani e bianchi nelle strutture partitiche del movimento. Tutto questo lo avvicinava politicamente alle posizioni assunte da altri vecchi dirigenti che erano entrati in contrasto con la direzione del Frelimo, tra cui Lázaro Nkavandame e Paulo Gumane. In

\footnotetext{
In verità, nonostante l'esistenza di una fazione apertamente contraria alla partecipazione di bianchi nelle strutture del Frelimo, prevalse una visione non razziale che accettava la partecipazione di tutti i mozambicani - indipendentemente dalla razza - nella lotta per l'indipendenza del Mozambico. Cfr. Passerini 1970.
} 
questo contesto, l'ascesa di Simango alla presidenza provocò la sfiducia di una parte significativa del Comitato Centrale del Frelimo. Così, nell'aprile del 1969, il Comitato Centrale decise di istituire un triumvirato presidenziale per gestire il movimento, costringendo Uria Simango a condividere il potere con altri due esponenti: Samora Machel, un ex-infermiere nero e leader militare della guerriglia, e Marcelino dos Santos, un intellettuale meticcio ed una delle figure di spicco della tendenza detta "rivoluzionaria". Questa situazione generò rapidamente attriti in seno alla direzione del Frelimo. Nel novembre del 1969, Uria Simango pubblicò un documento dando appoggio ai vecchi dissidenti del movimento ed accusando Samora Machel e diversi altri dirigenti di cospirare per ucciderlo. Infine, nel maggio del 1970, il Comitato Centrale decise di espellere Simango dal Frelimo. Samora Machel fu allora eletto Presidente del Frelimo, mentre Marcelino dos Santos prese il posto di Vice Presidente. A cominciare da questo momento, il Frelimo subì una chiara radicalizzazione a sinistra, il che portò all'allontanamento di un numero significativo di quadri ed antichi dirigenti più conservatori, soprattutto neri, che non vedevano di buon occhio la partecipazione di bianchi ed indiani nelle fila del movimento [Opello 1975, 62-82]. Rispetto a ciò, occorre sottolineare che la direzione del Frelimo rigettò sempre qualsiasi tentativo di razzializzazione del principio di nazionalità, mostrandosi aperta alla militanza di mozambicani di tutte le razze, bianchi inclusi [Bragança, Wallerstein 1982].

Oltre al Frelimo, dobbiamo rilevare l'esistenza di un nazionalismo mozambicano di matrice africana, nero, che non si riconosceva interamente nel Frelimo. Fu il caso dei gruppi di nazionalisti neri, originari in gran parte del centro-nord del Mozambico, che daranno vita al Comitato Rivoluzionario del Mozambico (Comité Revolucionário de Moçambique, COREMO), fondato a Lusaka, nel giugno del 1965. Sul piano militare, il COREMO non rappresentò mai un'alternativa valida al Frelimo e tanto meno costituì una vera minaccia al colonialismo portoghese. Sul piano politico, il COREMO incarnò anche un progetto nazionalista radicalmente differente da quello assunto dal Frelimo, nella misura in cui pretendeva di rappresentare esclusivamente le popolazioni colonizzate nere e non l'insieme delle popolazioni che formavano la totalità mozambicana e che includeva - oltre ai neri - i meticci e le minoranze di ori- 
Sul piano militare, la guerriglia fece notevoli progressi verso la fine del periodo coloniale, nonostante i portoghesi avessero lanciato una poderosa operazione militare nel 1970, denominata Nó Górdio, che però non era riuscita a sconfiggere le forze del Frelimo. Infatti, i guerriglieri avevano proceduto ad un ritiro strategico, dislocando le forze in altre aree e riprendendo poi a combattere con un'intensità ancora maggiore. Inoltre, la costruzione da parte dei portoghesi della gigantesca diga di Cabora Bassa, sul fiume Zambesi, aveva permesso alla guerriglia di disporre di un bersaglio militare mediaticamente rilevante, il che aveva obbligato il Portogallo a concentrare le truppe in questa zona per proteggere la costruzione dagli attacchi del Frelimo. Si faceva, d'altronde, sempre più evidente il logorio materiale e morale delle Forze Armate Portoghesi. Nel 1973, il massacro di un numero elevato di africani da parte delle truppe coloniali nella zona di Wiriyamu provocò una forte reazione internazionale, il che contribuì ad un maggior isolamento politico del regime coloniale, a grande beneficio del Frelimo [Hastings 1974].

Verso la fine del 1973, la situazione militare in Mozambico diventava sempre più difficile per i portoghesi. Il Frelimo aveva intensificato gli attacchi e le sue azioni erano giunte a meno di cento chilometri della città di Beira. La popolazione bianca, che fino ad allora era stata mal informata sulla reale situazione militare, cominciò a dar segni di forte apprensione politica per "l'inatteso" avanzamento del Frelimo. Infatti, una parte significativa della minoranza bianca temeva per il proprio futuro nel territorio ed aumentava sempre di più la sfiducia nell'operato dell'esercito portoghese, che era considerato poco efficace nel contrastare la guerriglia. Così, nel gennaio del 1974, a seguito della morte della moglie di un fazendeiro bianco durante un'azione del Frelimo, nella regione di Vila Pery, la popolazione bianca di Beira manifestò violentemente 
contro le truppe portoghesi, accusandole di non far nulla per eliminare la guerriglia [Carvalho 1991]. Quest'episodio aprì un'insanabile frattura tra la popolazione bianca e le Forze Armate e contribuì all'aumento esponenziale della tensione politica nella società coloniale.

Di fronte all'acutizzazione della situazione militare, Jorge Jardim, un importante uomo d'affari e carismatico leader politico bianco residente a Beira, tentò di trovare un'intesa con il Frelimo. Con l'appoggio del Presidente Banda, del Malawi, e grazie all'intermediazione del Presidente Kaunda, dello Zambia, Jardim riuscì ad ottenere una sorta di "intesa" con il Frelimo nel settembre del 1973. Denominata "Programma di Lusaka", questa "intesa" prevedeva l'indipendenza del Mozambico, nel quadro di una Comunidade Lusíada, e la partecipazione del Frelimo nella futura gestione politica ed amministrativa del paese, ma senza che la sua posizione (eventualmente dominante) diventasse "esclusiva". Parimenti veniva assicurata la protezione degli interessi economici portoghesi nella colonia ed il rispetto dei diritti della minoranza bianca ${ }^{6}$.

Munito di queste "garanzie", Jorge Jardim cercò allora di convincere il governo portoghese ad intavolare negoziati con il Frelimo. Ma l'ipotesi di una trattativa con la guerriglia fu rigettata da Marcelo Caetano [Souto 2007]. Per Caetano, il Frelimo non esprimeva la volontà della popolazione mozambicana e tanto meno mostrava di garantire la conservazione della matrice portoghese del Mozambico. Di fronte al rifiuto del capo del governo, Jardim cominciò a "pensare" di attuare la secessione della colonia con l'intenzione di negoziare da solo - cioè, senza la partecipazione del governo di Lisbona - con il Frelimo.

La rivoluzione del 25 aprile del 1974, innescata dal colpo di Stato organizzato dal Movimento das Forças Armadas (MFA) - il quale avrà

Public Record Office (PRO), FCO 9/2065, Relations Between Portugal and Africa, 1974 (British High Commission, Blantyre, 31/07/1974). Cfr. Jardim 1976, 119-120; Antunes 1996.

7 Sulla politica coloniale di Marcelo Caetano si veda: Souto 2007, Castilho 2012; Pimenta 2016, 12-30. 
un ruolo determinante nelle fasi finali del processo di indipendenza del Mozambico - cambiò completamente lo scenario. Tra le molteplici ragioni di sollevazione del MFA vi era anche la richiesta di porre fine alle guerre coloniali portoghesi e con esse all'impegno militare di tanti giovani.

La rivoluzione colse Jardim di sorpresa mentre si trovava in Portogallo, il che non gli permise di coordinare le attività politiche dei suoi sostenitori in Mozambico. Essendogli impedito di tornare nella colonia dal potere rivoluzionario, Jorge Jardim rimase inizialmente nella madrepatria come consigliere del Generale Spínola. Ma, di fronte al mandato di cattura emesso dalle autorità portoghesi, fuggì dapprima in Spagna e poi nell'Africa Australe, dove rimase muovendosi in continuazione tra il Malawi, lo Swaziland e il Sudafrica. E, nonostante i suoi tentativi di riallacciare i contatti con il Frelimo, finì per essere travolto dagli eventi, rinnegato sia dai portoghesi, che dai nazionalisti del Frelimo, che lo guardavano con qualche timore e molta diffidenza [Jardim 1976, 91135; Antunes 1996; Santos 2006].

Contemporaneamente sorse in Mozambico una "forza politica intermedia" tra il potere coloniale e il Frelimo. Si trattava del Grupo Unido de Moçambique (GUMO), che sebbene si presentasse come multirazziale, aveva una leadership essenzialmente nera. Il GUMO pare fosse stato promosso - o perlomeno appoggiato - dagli elementi più liberali del regime coloniale, specificatamente dal Ministro Baltazar Rebelo de Sousa, con la connivenza del Governatore Generale del Mozambico, l'ingegner Pimentel dos Santos. L'obiettivo dei suoi mentori era quello di rubare spazio politico al Frelimo e, al contempo, di formare una élite politica nera capace di governare il territorio dopo l'indipendenza. Si pensava, forse, all'istituzione di un governo di maggioranza nera, pur mantenendo l'economia saldamente nelle mani della minoranza bianca ed in stretta relazione com l'ex-metropoli. Il 25 aprile sorprese tuttavia il GUMO, che non era ancora sufficientemente organizzato per costi- 
tuirsi come valida alternativa al Frelimo ${ }^{8}$.

In questo senso, alla vigilia della rivoluzione in Portogallo, la situazione politica nella colonia era abbastanza tesa, essendo noto il degrado della situazione militare e l'allontanamento della popolazione bianca da parte delle Forze Armate Portoghesi. Era ugualmente percepibile l'apprensione della popolazione bianca per il proprio futuro. Tutto questo non presagiva la realizzazione di un tranquillo processo di transizione verso l'indipendenza.

\section{La caduta del regime in Mozambico}

Le prime notizie del golpe militare di Lisbona giunsero a Lourenço Marques la mattina del 25 aprile del 1974. Di fronte al golpe, il Governatore Generale, Pimentel dos Santos, abbozzò un tentativo di resistenza, avendo ordinato la chiusura dell'aeroporto della capitale. Tuttavia, l'intervento di un distaccamento di paracadutisti favorevoli alle forze rivoluzionarie disfece rapidamente qualsiasi velleità controrivoluzionaria da parte del Grupo dos Democratas de Moçambique. Manifesto del 25 aprile 1974, giorno della "Rivoluzione dei Garofani" in Portogallo. Foto di Ricardo Rangel, grafica di José Freire. Mozambico, 1974. Fonte: International Institute of Social History, call nr: BG E9/723.

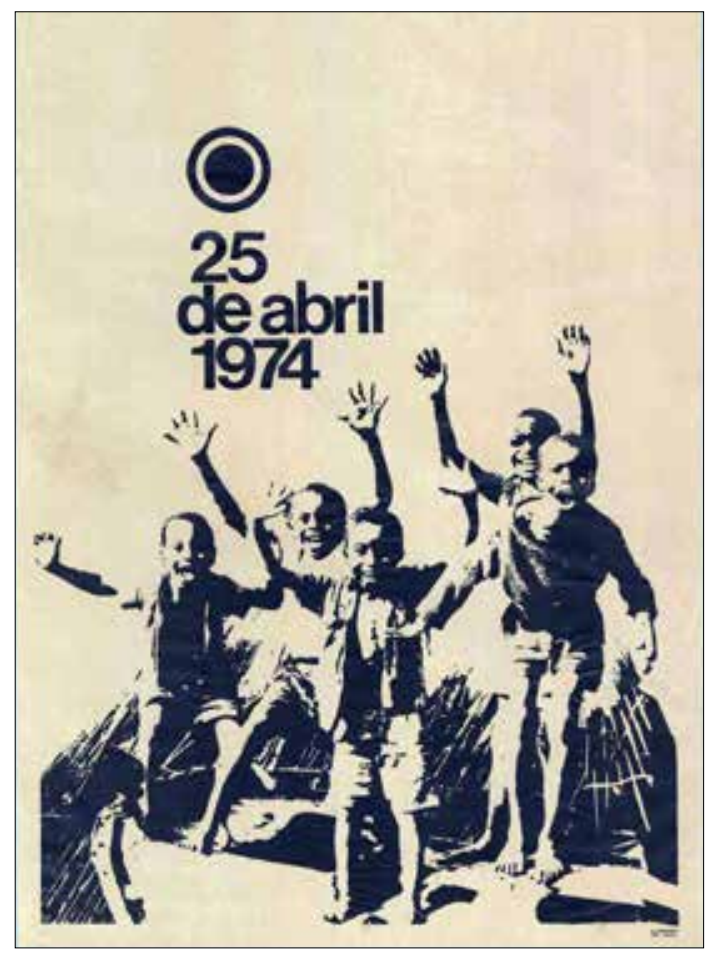

PRO, FCO 9/2049, Overseas Territories of Portugal, 1974; PRO, FCO 45/1533, Policy of Portugal to Her Overseas Territories, 1974. 
Governatore. Due giorni dopo, il Governatore Generale fu esonerato dalle sue funzioni, sostituito dal Segretario Generale, Coronel David Ferreira, in qualità di Incaricato dal Governo ${ }^{9}$. Immediatamente fu decretata la fine della censura per la stampa e la soppressione dell'Azione Nazionale Popolare, ma fu assicurata la continuazione della temuta Direzione Generale di Sicurezza (Direç̧ão Geral de Segurança, DGS, ex. PIDE), trasformata in "polizia di informazioni militari»"

Chiaramente, il 25 aprile suscitò un grande interesse nella società mozambicana, specialmente tra la popolazione bianca, che accompagnò l'evolversi del processo con curiosità e soddisfazione, ma anche con qualche timore per il proprio futuro nel territorio. A Lourenço Marques, a Beira e a Vila Pery si ebbero manifestazioni d'appoggio alla Giunta Militare (Junta de Salvação Nacional, JSN) capeggiata dal Generale Spínola. Il Generale António de Spínola, ex Governatore della Guinea, era un ufficiale di grande prestigio nell'esercito e nel paese. Nel gennaio 1974, Spínola era stato nominato Vice Capo di Stato Maggiore Generale delle Forze Armate, incarico che fu creato apposta per lui da Marcelo Caetano. Tuttavia il 22 febbraio del 1974, Spínola publicò il libro Portugal e o Futuro, nel quale denunciò l'impossibilità di una soluzione militare per la guerra coloniale e propose quindi il raggiungimento di una soluzione fondamentalmente politica. In altre parole, Spínola difese la fine della guerra e l'ottenimento di una soluzione politica che doveva passare attraverso il riconoscimento del diritto dei popoli delle colonie all'autodeterminazione. L'autodeterminazione, però, sarebbe stata raggiunta dopo un periodo di transizione, o meglio di preparazione, e tramite la realizzazione di un referendum. Spínola auspicava che tale referendum portasse alla costituzione di una Comunità di Stati di Lingua Portoghese, una specie di Commonwealth Lusitana. Il libro fu uno dei maggiori best sellers della storia editoriale portoghese e dette

9 «Notícias», n. 16.122, 28 aprile 1974, 1.

10 «Notícias», n. 16.124, 30 aprile 1974, 1. 
un enorme prestigio politico interno ed esterno a Spínola, compreso un significativo appoggio popolare. Ma la pubblicazione di Portugal e o Futuro provocò un enorme scandalo politico tra le fila del regime. Pressato dai settori più conservatori della dittatura, Marcelo Caetano fu "obbligato" a dimettere Spínola il 14 marzo 1974."

In nessuna delle tre manifestazioni venne affrontato il problema della guerra e la questione dell'indipendenza del Mozambico. Persino la stampa ebbe inizialmente una certa ritrosia a trattare in modo aperto la questione dell'indipendenza, optando per l'allineamento alla posizione ufficiale espressa da Spínola (1974). Così «Notícias», il principale quotidiano del Mozambico, pubblicò il Programma della JSN, dove si parlava di trovare una soluzione al "problema ultramarino", ma non si accennava alla questione dell'indipendenza ${ }^{12}$. Infatti, Spínola distingueva i principi dell'autodeterminazione e dell'indipendenza, per cui riconosceva il diritto dei popoli coloniali alla prima, ma difendeva il mantenimento dei territori africani sotto la bandiera portoghese attraverso la formazione di una federazione tra il Portogallo e le sue colonie. Federazione questa che sarebbe stata sottoposta a un referendum popolare ${ }^{13}$. Intanto, il Frelimo aveva reagito con soddisfazione alla caduta dell'Estado Novo, ben accogliendo la vittoria delle forze democratiche portoghesi. Ma, contemporaneamente, aveva messo bene in chiaro che non c'era un "colonialismo democratico", per cui l'unico modo per mettere la parola fine alla guerra era il riconoscimento portoghese del diritto del popolo mozambicano all'indipendenza. Così, il Frelimo rigettava parimenti l'idea di costituire una federazione col Portogallo ${ }^{14}$. Il 3 maggio 1974, la direzione del Frelimo decise di aumentare l’intensità della lotta

Dopo la caduta del regime, il Generale Spínola fu nominato Presidente della Repubblica dalla Giunta Militare nel maggio del 1974. Cfr. Rodrigues 2010.

12 «Notícias», n. 16.121, 27 aprile 1974, 2.

13 «Notícias», n. 16.122, 28 aprile 1974, 1.

14 «Notícias», n. 16.123, 29 aprile 1974, 1. 
armata contro le truppe portoghesi, in modo tale da sfruttare il clima di confusione generato dal golpe in Portogallo [MacQueen 1998, 159160].

D'altro canto, la fine della dittatura aveva permesso la libera costituzione - e la legalizzazione - dei movimenti politici. Rispetto a ciò, si assistette ad una proliferazione di partiti politici, molti dei quali senza rappresentatività tra la popolazione mozambicana. Ci furono però, come esamineremo nei paragrafi seguenti, alcuni movimenti che ebbero una partecipazione rilevante nel processo d'indipendenza.

\section{GUMO}

Il Grupo Unido de Moçambique (GUMO) fu il primo movimento a presentarsi alla popolazione, attraverso la pubblicazione di un manifesto, il 29 aprile del 1974. In questo manifesto, il GUMO sottolineava gli sforzi per costituirsi in "associazione civica" legalmente riconosciuta prima del 25 aprile. Il GUMO presentò anche il suo programma, il cui obiettivo principale era «l'ottenimento di un'autonomia politica progressiva, dentro le istituzioni politiche vigenti nello Spazio Portoghese venendo incontro alle nuove aperture». Quest'autonomia doveva essere ottenuta con la partecipazione di tutti i mozambicani e secondo i principi di «multirazzialità, dialogo e rispetto dei parametri legali legittimamente stabiliti». Il GUMO si proponeva di servire gli interessi di una Comunità Luso-Mozambicana e, soprattutto, di lottare per la partecipazione attiva di «neri-meticci» nella struttura economica del Mozambico. Rispetto a ciò, il GUMO considerava che l'indipendenza politica potesse essere effettiva solo con l'indipendenza economica. Il GUMO presentò infine i nomi dei dirigenti che formavano la sua Commissione Centrale, tra cui si distinguevano Máximo Dias (Presidente, indiano), Joana Simeão (Vice Presidentessa, nera) e Jorge de Abreu (Responsabile delle Attività 
Economiche, bianco) $)^{15}$. Tuttavia, sebbene lottasse per la multirazzialità, il GUMO si presentava in sostanza come il difensore degli interessi della maggioranza nera. Questa situazione faceva del GUMO una specie di concorrente interno del Frelimo, poichè entrambi cercavano appoggi all'interno della stessa base sociale: la maggioranza africana' ${ }^{16}$. La legalizzazione del GUMO generò alcune tensioni politiche già nei primi giorni del mese di maggio del 1974. Questa tensione fu percepibile nel primo comizio realizzato da questo movimento nello stadio di Xipamanine, a Lourenço Marques, il 3 maggio. Il comizio radunò circa 20.000 persone, soprattutto neri provenienti dai sobborghi, anche se non tutti erano lì per appoggiare il GUMO. Durante il discorso di Joana Simeão, una parte significativa dei presenti si pronunciò contro il GUMO, appoggiando invece il Frelimo. Si generò allora un certo caos e la situazione divenne talmente tesa che Joana Simeão dovette ricorrere all'aiuto della polizia per lasciare il comizio ${ }^{17}$. L'episodio fu la dimostrazione delle difficoltà di mobilitazione politica da parte del GUMO, su cui pesava in questo caso la concorrenza del Frelimo, che disponeva di un prestigio di molto maggiore tra la popolazione.

Parallelamente, alcune frange della minoranza bianca si confrontarono con i sostenitori del GUMO. Questi contraddittori furono particolarmente aspri a Beira, in occasione di un comizio svoltosi il 12 maggio del 1974. Il comizio degenerò in gravi incidenti con la popolazione bianca. Seguì una seconda manifestazione, questa volta di bianchi, che coinvolse migliaia di persone, di fronte alla residenza del Governatore, e davanti al Generale Costa Gomes' ${ }^{18}$, che era in visita in città. I manifestanti chiedevano alle autorità portoghesi che mettessero fine alle

\footnotetext{
15 «Notícias», n. 16.123, 29 aprile 1974, 2.

16 "Notícias», n. 16.125, $1^{\circ}$ maggio 1974, 2.

17 «Notícias», n. 16.127, 4 maggio 1974, 1-2.

18 Il Generale Costa Gomes era il numero due della Giunta Militare e succedette a Spínola come Presidente della Repubblica il 30 settembre 1974.
} 
violenze e che fossero presi provvedimenti affinché venissero garantite la pace e la sicurezza della popolazione. In tutta risposta, Costa Gomes chiese ai manifestanti di calmarsi, ma davanti all'accrescersi della tensione, le forze di sicurezza intervennero, scontrandosi coi manifestanti ${ }^{19}$. Gli incidenti di Beira provocarono l'allontanamento quasi totale dei bianchi dal GUMO, anche di quegli imprenditori che avrebbero patrocinato il movimento. Così, il partito entrò in un processo di rapida dissoluzione politica, con l'abbandono di una parte della cupola dirigente. Jorge de Abreu fu il primo a rendere pubbliche le sue dimissioni dalla Commissione Centrale ${ }^{20}$. Ne seguì una specie di "guerra interna” tra Máximo Dias e Joana Simeão. Quest'ultima rivendicava un'identificazione politica con la popolazione nera, in particolare con il popolo Macua, che costituiva il maggior gruppo etnico-linguistico del Mozambico. Alla metà di giugno, Joana Simeão provocò una scissione all'interno del GUMO e costituì un nuovo movimento denominato Fronte Comune del Mozambico (Frente Comum de Moçambique, FRECOMO ${ }^{21}$. Intanto, Máximo Dias annunciò la fine di tutta l'attività politica del GUMO a partire dal 10 luglio 1974, pensando che «la creazione di una terza forza era soltanto uno stratagemma per imporre il neocolonialismo in Mozambico ${ }^{22}$. L'esperienza politica rappresentata dal GUMO era giunta al suo termine, risolvendosi in un fallimento.

\section{Democratici del Mozambico}

Un altro "partito" con un notevole peso politico fu il movimento dei Democratici del Mozambico. Lo zoccolo duro del movimento era for-

19 «Notícias», n. 16.136, 13 maggio 1974, 1 e 5.

20 «Notícias», n. 16.154, 31 maggio 1974, 1.

21 «Notícias», n. 16.185, 3 luglio 1974, 12.

22 «Notícias», n. 16.187, 5 luglio 1974, 1. 
mato da "vecchi" oppositori, soprattutto avvocati e liberi professionisti bianchi, tra i quali Almeida Santos, Pereira Leite, Adrião Rodrigues, Rui Baltazar, etc. Dopo la rivoluzione del 25 aprile, questi oppositori avevano costituito una sorta di movimento di pressione politica sul regime coloniale e di appoggio al Frelimo. Così, i Democratici consideravano che la soluzione al problema mozambicano era di «natura politica e non militare». Una soluzione che doveva essere «l'espressione della reale volontà del popolo del Mozambico liberamente autodeterminato»²3. Pubblicato il $1^{\circ}$ maggio 1974, il "Manifesto dei Democratici Mozambicani" ricevette l'appoggio di circa tremila persone, radunatesi nell'Aula Magna dell'Università di Lourenço Marques ${ }^{24}$. Il movimento, sorto dapprima nella capitale, finì per ramificarsi in vari punti della colonia, dove sorsero gruppi autonomi di democratici, soprattutto a Beira e Nampula. Tutti questi gruppi si fusero in un'unica struttura il 27 maggio 1974, sotto la designazione di Democratici del Mozambico.

Tutti i gruppi di democratici accettavano i seguenti principi: a) non costituire, nè avere come obiettivo la costituzione di un partito politico; b) riconfermare il proprio appoggio all'MFA (Movimento das Forças Armadas) il movimento militare di della rivoluzione del 25 aprile 1974; c) considerare fondamentale la realizzazione di negoziati tra il governo portoghese e il Frelimo basati sul "riconoscimento del diritto del Popolo del Mozambico all'indipendenza"; d) denunciare tutti i tentativi di creazione di terze forze politiche. Oltre a ciò, i Democratici definirono come obiettivi: 1) spingere lo smantellamento del sistema creato dal "fascismo-colonialismo"; 2) chiarire e rendere cosciente politicamente la popolazione; 3) far conoscere e spiegare il programma del Frelimo alla popolazione; 4) impedire la rottura di unità politica del popolo del Mozambico $^{25}$.

23 «Notícias», n. 16.125, 1 maggio 1974, 2 e 13.

24 «Notícias», n. 16.126, 3 maggio 1974, 1 e 2.

25 «Notícias», n. 16.150, 27 maggio 1974, 1. 
Dall'altro lato, i Democratici riuscirono ad influenzare il governo portoghese nella scelta del nuovo Governatore, ottenendo la nomina di uno dei suoi membri, l'avvocato Soares de Melo, che ebbe l'incarico nel giugno del 1974. In questo, i Democratici furono molto aiutati dalla nomina di Almeida Santos - uno dei fondatori del movimento - come Ministro del Coordinamento Interterritoriale, del 1. ${ }^{\circ}$ Governo Provvisorio, il 15 maggio 197426. Questo Ministero succedette al Ministero dell'Oltremare, ereditandone la struttura e parte delle sue funzioni. Almeida Santos era stato scelto, per l'esercizio di queste funzioni, in virtù dei suoi antichi legami con l'opposizione democratica nella metropoli, ma anche per la sua conoscenza della realtà africana [Santos 1975; Santos 2006].

In questo contesto, i Democratici del Mozambico cominciarono a fare pubblicamente campagna a favore del Frelimo, con la connivenza delle autorità portoghesi. Il 26 maggio, il Dr. Adrião Rodrigues dichiarò che i Democratici sottoscrivevano interamente il programma del Frelimo ${ }^{27}$. Il 31 maggio, in una sessione svoltasi a Inhambane, i Democratici sottoscrissero che il Frelimo era l'unico movimento rappresentativo del Mozambico, fatto che toglieva ogni legittimità alle altre forze politiche mozambicane ${ }^{28}$. Il giorno seguente, Soares de Melo, ai microfoni di Radio Club del Mozambico, dichiarò che il socialismo era la miglior soluzione per il paese, facendo così una chiara allusione all'orientamento ideologico del Frelimo ${ }^{29}$. Il 4 giugno 1974, Adrião Rodrigues dichiarò che il regime del partito unico poteva non essere un fatto negativo, accettando questa ipotesi per il Mozambico, purché questo governo fosse formato dal Frelimo. Al contrario, l'esistenza di vari partiti poteva

\footnotetext{
26 «Notícias», n. 16.139, 16 maggio 1974, 1.

27 «Notícias», n. 16.149, 26 maggio 1974, 18.

28 «Notícias», n. 16.155, 1 giugno 1974, 4.

29 «Notícias», n. 16.156, 2 giugno 1974, 1.
} 
costituire un pericolo, nella misura in cui poteva «disunire il popolo» ${ }^{30}$. I Democratici del Mozambico riuscirono a catturare l'appoggio di una parte significativa dei settori più progressisti della comunità bianca. Però, il loro discorso radicale, di severa critica al sistema capitalista e con riferimenti al marxismo, così come il rifiuto a riconoscere la legittimità di altre forze politiche che non fosse il Frelimo, con la quale reclamavano una totale identificazione politico-ideologica, finì per spaventare e, conseguentemente, allontanare la maggioranza della popolazione bianca. In questo modo, i Democratici del Mozambico, che volevano preparare la minoranza bianca all'indipendenza, canalizzando il suo appoggio verso il Frelimo, finirono per produrre l'effetto contrario, perlomeno su questo punto, alienando - più che mobilitando - le eventuali simpatie dei bianchi.

\section{FICO}

Sul fronte opposto, sorse un altro movimento politico denominato FICO. Fondato il 5 maggio 1974, il FICO mobilitò i settori più conservatori della popolazione bianca, inizialmente con il nome di Fronte Indipendente di Convergenza Occidentale (Frente Independente de Convergência Ocidental). Per il FICO, il Mozambico era - e doveva continuare ad essere - "terra portoghese». Il FICO rifiutava anche il socialismo sovietico, per cui sosteneva l'adesione al campo occidentale nell'ambito della lotta tra i due blocchi della Guerra Fredda. Infine, il FICO considerava il Frelimo una forza politico-militare nemica, perlomeno finché muoveva guerra al Portogallo. Svariate migliaia di bianchi parteciparono alla prima manifestazione del FICO a Lourenço Marques $^{31}$. Tuttavia, il FICO tardò qualche tempo a chiarire la sua posizione

\footnotetext{
30 «Notícias», n. 16.159, 5 giugno 1974, 2.

31 «Notícias», n. 16.129, 6 maggio 1974, 1 e 4.
} 
sia verso il processo rivoluzionario che verso la questione dell'indipendenza. Inizialmente fece intendere che rifiutava l'idea d'indipendenza. Ma subito, il 7 maggio, il FICO fece sapere che appoggiava il programma della Junta de Salvação Nacional (JSN). Dieci giorni dopo, il FICO apparve già con uno slogan politicamente meno aggressivo: Ficar Convivendo («Rimanere Convivendo»), designazione che venne adottata come nome definitivo il 21 maggio. Il FICO dichiarò anche che il principale obiettivo del movimento era la "permanenza del Mozambico in seno alla Comunità Luso-Brasileira» ${ }^{32}$, accettando l'idea di un referendum, in linea con la proposta di Spínola, affinchè venisse ascoltata la volontà dei «Popoli del Mozambico»"

\section{La Convergenza Democratica di Beira}

Intanto, a Beira era sorta un'organizzazione di bianchi liberali designata Convergenza Democratica del Mozambico (Convergência Democrática de Moçambique, CDM). Tenendo come figura trainante João Soares Cardoso, la Convergenza Democratica si collocava politicamente tra il FICO ed i Democratici del Mozambico, rivendicando una posizione intermedia ed un'identificazione con i valori della social-democrazia. La Convergenza Democratica difendeva l'istituzione di un regime democratico, dove tutte le tendenze ideologiche potessero esprimere liberamente la loro opinione e partecipare al governo del paese dopo l'indipendenza secondo la volontà del popolo espressa in libere elezio$\mathrm{ni}^{34}$. Era pertanto a favore dell'elezione democratica dei rappresentanti del popolo mozambicano, manifestandosi contraria a qualunque tipo

\footnotetext{
32 «Notícias», n. 16.142, 19 maggio 1974, 4.

33 «Notícias», n. 16.158, 4 giugno1974, 5.

34 «Notícias», n. 16.161, 7 giugno 1974, 4.
} 
di dittatura ${ }^{35}$. Dall'altro lato, la Convergenza Democratica riconosceva il ruolo centrale del Frelimo nella lotta contro il colonialismo, così come il suo diritto a partecipare al processo d'indipendenza, ma non accettava che il movimento capeggiato da Samora Machel fosse considerato l'unico rappresentante del popolo mozambicano. In effetti, la Convergenza Democratica considerava che il Frelimo stava negando al popolo mozambicano il diritto all'autodeterminazione, nella misura in cui esigeva di essere l'unico interlocutore nei negoziati. Una situazione che poteva portare all'istituzione di un regime totalitario in Mozambico. Ora, la posizione della Convergenza Democratica collideva frontalmente con la posizione assunta dai Democratici del Mozambico, che non concepivano l'esistenza di un'altra forza politica rappresentativa del popolo mozambicano che non fosse il Frelimo.

Però, fu intorno al controllo del giornale "Notícias» da Beira che si ebbe il principale scontro politico tra i due movimenti. Infatti, durante il mese di maggio del 1974, i Democratici del Mozambico erano riusciti ad ottenere il controllo dei principali mezzi d'informazione di Lourenço Marques, specificatamente del giornale «Notícias» e della Radio Club del Mozambico. A Beira la situazione era stata diversa. I Democratici si erano mostrati incapaci di ottenere il controllo del giornale «Notícias» da Beira, rendendosi necessario l'intervento diretto delle autorità portoghesi affinchè la direzione del giornale fosse tolta al circolo politico di Jorge Jardim, che era in esilio. L'amministrazione del «Notícias» da Beira si trovò allora a carico del Governo Generale ${ }^{36}$. Ma, all'inizio di luglio del 1974, la Convergenza Democratica ottenne da parte del Governo l'autorizzazione a gestire il «Notícias» da Beira. Questa situazione dispiacque profondamente ai Democratici, che criticarono la decisione del Governo ed accusarono la Convergenza Democratica di essere l'espressione politica del grande capitale finanziario. I due movimenti

\footnotetext{
35 «Notícias», n. 16.174, 22 giugno 1974, 7.

36 «Notícias», n. 16.171, 19 giugno 1974, 1.
} 
iniziarono allora una specie di "guerra dei comunicati", con scambio di accuse ed attacchi verbali che durarono settimane. Tuttavia, i Democratici riuscirono a mobilitare a loro favore i militari portoghesi, avendo la Commissione Regionale dell'MFA a Beira espresso parere contrario alla consegna del giornale alla Convergenza Democratica ${ }^{37}$. Così, sotto la pressione dell'MFA, le autorità portoghesi tornarono sui propri passi, rifiutandosi di consegnare la gestione del giornale alla Convergenza Democratica $^{38}$.

I Democratici del Mozambico riuscirono anche ad influenzare i militari portoghesi affinchè procedessero alla neutralizzazione politica della Convergenza Democratica. Infatti, il 28 luglio del 1974, a seguito di una riunione con la Commissione Coordinatrice dell'MFA, la Convergenza Democratica annunciò la sospensione delle sue attività. In un ultimo comunicato rivolto alla popolazione, la Convergenza Democratica denunciò l'estremismo di chi si era «sottratto alla discussione dei problemi», affermando l'esistenza di un «controllo ideologico» sulla stampa ed in radio da parte dei gruppi politici interessati a promuovere sistematicamente l'immagine del Frelimo e a negare e denigrare punti di vista divergenti ${ }^{39}$.

\section{Insuccesso del Governo Provvisorio di Soares de Melo}

Nel frattempo, la crescita della tensione politica e sociale si risolse nella comparsa di partiti estremisti e finanche a carattere militare, specificatamente l'AMA, organizzazione che ricorreva alla violenza per esprimere la sua opposizione ai Democratici e al Frelimo, e che fu protagonista di

37 «Notícias», n. 16.204, 22 luglio 1974, 1.

38 «Notícias», n. 16.208, 27 luglio 1974, 1.

39 «Notícias», n. 16.210, 29 luglio 1974, 5. 
alcuni attentati con granate a Lourenço Marques ${ }^{40}$.

La comparsa di atti di violenza fu concomitante allo svuotamento di autorità del governo coloniale in Mozambico. Infatti, il processo rivoluzionario provocò l'indebolimento dell'apparato poliziale e militare sul quale poggiava l'amministrazione portoghese. In assenza di direttive chiare da parte di Lisbona e non disponendo di una linea direttiva ben definita rispetto al processo di decolonizzazione, le autorità portoghesi andarono gradualmente perdendo il controllo sulla situazione politica nella colonia, il che ebbe evidenti conseguenze sulla questione dell'ordine e della sicurezza. In termini militari, l'esercito portoghese cessò ogni attività offensiva, rispondendo appena al fuoco nemico, il che facilitò la penetrazione di guerriglieri nel territorio mozambicano, così come la formazione di gruppi armati isolati.

D'altro canto, il Governo Provvisorio di Soares de Melo non ebbe tempo nemmeno di attuare misure concrete che attennuassero la tensione politica e sociale, dal momento che rimase in funzione meno di due mesi. Di fatto, Soares de Melo tentò di assumere una posizione di compromesso tra gli orientamenti di Spínola e le pressioni dei Democratici del Mozambico. Di fatto, Soares de Melo cercò di andare incontro ad alcune rivendicazioni dei Democratici, in particolare in quelle che riguardavano lo smantellamento dell'apparato repressivo della dittatura. Così, il Governatore si preoccupò di eliminare l'Organizzazione Provinciale dei Volontari di Difesa Civile (Organização Provincial de Voluntários de Defesa Civil, OPVDC) e tutte le milizie paramilitari esistenti sul territorio mozambicano ${ }^{41}$. E, l'8 giugno 1974, fu lanciata un'operazione militare di cattura degli agenti dell'estinta PIDE/DGS, la cosiddetta Operazione Zebra. Nell'azione furono imprigionati circa duecento agenti della polizia politica, il che ridusse significativamente

40 «Notícias», n. 16.176, 24 giugno 1974, 1; «Notícias», n. 16.177, 25 giugno 1974, 2.

41 «Notícias», n. 16.169, 17 giugno 1974, 1. 
la rete di informazione militare delle Forze Armate Portoghesi. Infine, il 17 giugno 1974, il Governo Generale emanò un mandato di cattura contro Jorge Jardim, accusandolo di essere il mentore della "reazione» in Mozambico ${ }^{42}$.

Però, queste misure non furono sufficienti per soddisfare i Democratici del Mozambico, che pretendevano un risanamento totale dell'amministrazione pubblica, non accettando al contempo l'esistenza di altre forze politiche all'infuori del Frelimo. Ora, la decisione del Governo di consegnare la gestione del giornale «Notícias» da Beira alla Convergenza Democratica provocò - come visto - una fortissima reazione dei Democratici, che non intendevano - e non accettavano - la posizione di compromesso assunta dal Governatore Generale. Per questo, i Democratici cominciarono a criticare Soares de Melo, per la sua accondiscendenza verso le altre forze politiche ${ }^{\star 3}$.

Tuttavia, furono fattori esterni alla colonia che portarono alla caduta precipitosa del Governo Provvisorio del Mozambico. Di fronte all'aggravamento dell'escalation di violenza in Angola, Spínola nominò una Giunta Governativa per l'Angola, con connotazioni militari, presieduta dall'Ammiraglio Rosa Coutinho, il 24 luglio 1974. Per emulazione politica, fu annunciata dalle autorità metropolitane la decisione di creare una Giunta Governativa per il Mozambico ${ }^{4}$. Di tutta risposta, Soares de Melo, sentendosi politicamente abbandonato da Lisbona, presentò le sue dimissioni il 25 luglio del 1974 ${ }^{45}$. Rimase tuttavia in carica, ancorché dimissionario, ancora qualche settimana, ma ormai senza nessun intervento significativo nel processo politico ${ }^{+6}$.

\footnotetext{
42 «Notícias», n. 16.170, 18 giugno 1974, 1.

43 «Notícias», n. 16.187, 5 luglio 1974, 5.

44 «Notícias», n. 16.207, 26 luglio 1974, 1.

45 «Notícias», n. 16.207, 26 luglio 1974, 1.

46 Alla metà di agosto del 1974, Soares de Melo trasmise formalmente i poteri al Segretario di Stato dell'Amministrazione Territoriale, Jorge Ferro Ribeiro, che assunse
} 


\section{Primi negoziati con il Frelimo}

Intanto, il Portogallo e il Frelimo avevano fatto i primi passi per giungere ad un accordo di cessate il fuoco. Così, nella seconda settimana di maggio del 1974, il Generale Costa Gomes dette ordine alle forze portoghesi di interrompere tutte le operazioni offensive e di assumere una posizione meramente difensiva e di protezione dei civili. Questa decisione fu presa quando la guerriglia aveva deciso di aumentare il suo sforzo bellico contro le truppe portoghesi, il che aveva facilitato significativamente le attività militari del Frelimo. Dall'altro lato, Costa Gomes fece appello al Frelimo perché accettasse un cessate il fuoco e si trasformasse in un partito politico. Così, inviò, ancora in quel mese, una delegazione di sei vecchi prigionieri politici a Dar-es-Salaam con una proposta di cessate il fuoco rivolta alla direzione del Frelimo [Spínola 1978, 292-294].

Ma i primi colloqui ufficiali ebbero luogo il 5/6 giugno, a Lusaka. In questi primi colloqui, Mário Soares e Samora Machel discussero le condizioni per stabilire un cessate il fuoco, avendo il Frelimo posto tre condizioni essenziali: 1) il riconoscimento del diritto all'indipendenza immediata del Mozambico; 2) il riconoscimento del Frelimo come unica legittima rappresentante del popolo mozambicano; $3^{\circ}$ ) il trasferimento del potere nelle mani del Frelimo. Ora, queste esigenze erano contrarie alle idee di Spínola, che pretendeva si organizzasse un referendum per decidere il futuro delle colonie portoghesi. Per questo, non fu più possibile procedere coi colloqui, che si bloccarono [MacQueen 1998, 164-167].

Tuttavia, il degrado della situazione militare nella colonia e, soprattutto, la pressione dell'MFA portarono ad un cambiamento della posizione portoghese. Infatti, alcune unità militari portoghesi cominciarono a negoziare accordi separati di cessate il fuoco con i guerriglieri, spe-

le funzioni di Incaricato del Governo. Cf. «NNotícias»», n. 16.231, 20 agosto 1974, 3. 
cificatamente a Cabo Delgado, in giugno, e a Tete, nel luglio 1974. Successivamente, la guarnigione militare portoghese di Omar, al confine con la Tanzania, si arrese al Frelimo ed i soldati portoghesi furono fatti prigionieri ed evacuati in Tanzania. Fu un avvenimento con un'enorme ripercussione mediatica e che abbatté ancora di più il morale delle truppe. Dall'altro lato, l'MFA andò acquisendo forza all'interno dell'apparato dello Stato coloniale, costituendosi in una specie di potere parallelo al Governo Provvisorio. Così, il $1^{\circ}$ luglio, l'MFA esigette la ripresa dei colloqui con il Frelimo e, dieci giorni dopo, raccomandò, in un memorandum, il riconoscimento del diritto all'indipendenza e del Frelimo come il più valido rappresentante del popolo del Mozambico. Il 20 luglio, in un'assemblea plenaria a Nampula, i delegati dell'MFA di Tete e di Cabo Delgado esigettero la realizzazione di un accordo con il Frelimo e, due giorni dopo, l'MFA ribadì la necessità di intavolare nuovi negoziati con il movimento guerrigliero. Il 23 luglio, il Governatore Generale del Mozambico informava Lisbona che le commissioni dell'MFA di Cabo Delgado e di Tete avevano emesso un ultimatum nel quale minacciavano di dichiarare unilateralmente il cessate il fuoco generale nel caso in cui il governo portoghese non lo avesse negoziato entro la fine del mese [MacQueen 1998, 161].

\section{La Legge Costituzionale n. 7/74, l'MFA ed i Democratici del Mozambico}

Tutti questi avvenimenti ebbero un peso significativo nell'evoluzione del processo politico portoghese, indebolendo considerevolmente la posizione di Spínola. In questo senso, davanti al rapido degrado della situazione militare e di fronte alla rilevante crescita delle pressioni del Movimento das Forças Armadas, il Presidente della Repubblica fu obbligato a fare modifiche nella politica coloniale per arrivare ad un rapido accordo con i guerriglieri nazionalisti. Così, il 26 luglio 1974, Spínola 
promulgò una nuova Legge Costituzionale, la Legge n. 7/74. La nuova Legge Costituzionale riconobbe «il diritto all'autodeterminazione, con tutte le sue conseguenze, inclusa l'accettazione dell'indipendenza dei territori ultramarini». Si trattava di una chiara vittoria dell'MFA, che difendeva la negoziazione diretta dell'indipendenza con le guerriglie nazionaliste, l'istituzione di un cessate il fuoco ed un rapido trasferimento di potere esclusivamente nelle mani di questi stessi movimenti [Pimenta 2010, 139-140].

Intanto, il Portogallo e il Frelimo effettuarono un nuovo ciclo di colloqui in vista di un rapido negoziato sull'accordo d'indipendenza, anche senza la preventiva realizzazione di un cessate il fuoco. In effetti, la promulgazione della legge n. $7 / 74$ aveva aperto la porta ad una rapida soluzione del problema della decolonizzazione, del che approfittò subito il Frelimo. Così, il Frelimo propose la realizzazione di negoziati con i portoghesi, a Dar-es-Salaam, tra il 30 luglio ed il 2 agosto 1974. La delegazione portoghese fu capeggiata da un ufficiale dell'MFA, Melo Antunes. L'incontro permise di stabilire il quadro generale del futuro processo d'indipendenza. Restò così deciso: 1) la realizzazione dell'indipendenza senza un previo referendum; 2) il riconoscimento del Frelimo come unico legittimo rappresentante del popolo del Mozambico; 3) il trasferimento del potere al Frelimo dopo un periodo di governo di transizione. In fondo, questa prima intesa tra le due parti rappresentava una chiara vittoria dei nazionalisti mozambicani, che vedevano le loro condizioni accettate dai portoghesi. Dal 14 al 17 agosto del 1974, ebbe luogo un nuovo giro di negoziati a Dar-es-Salaam, che confermò le posizioni precedentemente assunte. Erano state così messe le basi per la celebrazione dell'accordo finale [MacQueen 1998, 178-179].

Nel frattempo, sfruttando un nuovo contesto politico-militare a loro nitidamente favorevole, i Democratici del Mozambico conclusero la loro manovra di controllo dell'informazione, allineando i principali organi di comunicazione sociale esclusivamente con le posizioni del Frelimo. Questo allineamento portò al silenzio giornalistico tutti gli altri 
gruppi politici. Di fatto, i giornali smisero di pubblicare punti di vista, comunicati ed attività degli altri partiti. Invece, sulla stampa si passò a difendere la tesi che il Frelimo era «la legittima rappresentante del popolo mozambicano e che il potere doveva essere trasferito in regime di monopolio a questo Fronte»"

Oltre a ciò, una ben orchestrata campagna giornalistica procedette alla demonizzazione politica di tutti coloro che si opponevano al trasferimento di potere diretto ed esclusivo nelle mani del Frelimo e, quindi, alla creazione di un regime di partito unico. Così, i Democratici e la stampa pro-Frelimo etichettarono come «reazionari» e "colonial-fascisti» tutti coloro che esprimevano opinioni diverse da quelle difese dal movimento guerrigliero, in particolare coloro che pretendevano una consulta della popolazione mozambicana. Questa campagna giornalistica contro gli oppositori del Frelimo provocò forti critiche da parte dei vari settori della società mozambicana, in particolare tra la popolazione bianca più conservatrice, tra la minoranza indiana e finanche tra alcuni segmenti delle classi medie meticcia e nera ${ }^{18}$. Gruppi più radicali, scontenti dell'atteggiamento della stampa, arrivarono addirittura ad attaccare con l'esplosivo i giornali «Notícias» e $A$ Tribuna all'alba del 15 agosto ${ }^{19}$.

Tutto questo ebbe pesanti ripercussioni psicologiche sulla popolazione, generando insicurezza, confusione e paura, soprattutto tra la minoranza bianca, che non era preparata ad affrontare la nuova situazione politica creata dal processo di decolonizzazione e che temeva per il proprio futuro nel paese dopo l'indipendenza.

Quindi, durante il mese di agosto del 1974, si assistette alla radicalizzazione del discorso politico, all'estremizzazione degli antagonismi ed

\footnotetext{
47 «Notícias», n. 16.208, 27 luglio 1974, 1.

48 «Notícias», n. 16.225, 13 agosto 1974, 1. Cfr. Saavedra R. 1975; Ruas H. B. (s/d); Aguiar L. 1977.

49 «Notícias», n. 16. 228, 17 agosto 1974, 1.
} 
all'aumento generalizzato della tensione politica e sociale. Del resto, una raffica di scioperi, manifestazioni e proteste spazzarono il territorio, il che ebbe gravi ripercussioni sull'economia e finanche sull'approvvigionamento di generi di prima necessità nelle principali città. Il crimine e la violenza contro beni e persone crebbero in maniera esponenziale, generando un clima di forte insicurezza. Con l'esercito praticamente paralizzato, gruppi di banditi sparsero il panico tra le popolazioni rurali dei distretti del Centro e del Nord, causando numerose vittime tra neri, bianchi, meticci ed indiani, con i negozi (cantinas) di questi ultimi bersaglio preferenziale di assalti, ruberie e distruzioni. Nell'agosto del 1974, si ebbe addirittura un tentativo di "pulizia etnica" da parte di gruppi di africani armati contro le minoranze bianca ed indiana nella regione compresa tra Quelimane e António Enes e, più in generale, nella zona di Nampula ${ }^{50}$. Il Frelimo negò qualsiasi coinvolgimento negli accadimenti e mai si riuscì ad appurare con certezza chi c'era dietro questa "ondata di violenza anti-bianca». Ma il fatto è che la paura si impossessò di gran parte della popolazione bianca, in un modo tale che, tutte le settimane, cominciarono ad andarsene dalla colonia circa un migliaio di bianchi. Era l'inizio dell'esodo.

\section{Accordo di Lusaka}

Fu dunque in questo contesto di accentuato scontro politico e di forte tensione sociale che venne firmato l'accordo d'indipendenza del Mozambico, a Lusaka, il 7 settembre 1974. L'accordo di Lusaka stabilì il riconoscimento portoghese dell'indipendenza del Mozambico ed il trasferimento di poteri esclusivamente nelle mani del Frelimo, fissando la data dell'indipendenza al 25 giugno 1975 [Moita 1984, 289]. Fu

«Notícias», n. 16.222, 10 agosto 1974, 1; «Notícias», n. 16.225, 13 agosto 1974, 1-2; «Notícias», n. 16.227, 15 agosto 1974, 1. 
dichiarato un cessate il fuoco immediato. Tutti i prigionieri di guerra dovettero essere liberati e le forze militari portoghesi dovettero essere gradualmente concentrate nelle zone urbane al fine di facilitarne il rimpatrio. L'Accordo definì anche la costituzione di un Governo di Transizione, formato da sei ministri nominati dal Frelimo e tre dal Portogallo. Il Governo di Transizione sarebbe stato guidato da un Primo Ministro indicato dal Frelimo (Joaquim Chissano), mentre il Portogallo avrebbe nominato un Alto Commissario, le cui funzioni sarebbero state simili a quelle di un Capo di Stato, senza però una qualche autorità diretta sul Governo di Transizione. Con l'approvazione del Frelimo, Vitor Crespo fu nominato Alto Commissario [MacQueen 1998, 181-182].

L'Accordo non definì il sistema politico del nuovo paese, né tanto meno garantì l'insediamento di una democrazia multipartitica, attribuendo la prerogativa della decisione al Frelimo [Moita 1984, 289]. L'Accordo non salvaguardò neanche i diritti della minoranza bianca, nemmeno il diritto alla nazionalità mozambicana. Di fatto, molte questioni rimasero irrisolte, tra le quali i diritti di proprietà e la sicurezza dei cittadini portoghesi residenti in Mozambico, così come la ripartizione delle responsabilità finanziarie tra il Portogallo ed il nuovo Stato. Questioni che poi verranno a pesare in modo significativo durante il periodo di transizione ed in quello post-indipendenza. In questo senso, l'accordo di Lusaka servì appena a sanzionare a livello internazionale la consegna del Mozambico al Frelimo. I firmatari dell'Accordo giustificarono questa posizione col fatto che il Frelimo era stato l'unico movimento mozambicano ad acquisire una «legittimità popolare per la via rivoluzionaria», cioè quella militare. Per questo, erano state escluse dal processo d'indipendenza e rese illegali tutte le altre forze politiche mozambicane ${ }^{51}$.

51 O Acordo de Lusaka inconstitucional e antidemocrático não defende os interesses de Portugal e dos povos de Moçambique (1974), «Jornal Português de Economia \& Finanças», n. 325 , dal 16 al 31 dicembre, 7-12. 


\section{La rivolta di Lourenço Marques}

L'Accordo di Lusaka provocò una reazione immediata delle forze politiche escluse dal processo d'indipendenza (FICO, $\mathrm{PCN}^{52}$, etc.). Dirigenti politici bianchi - Gomes dos Santos (FICO), Hugo Velez Grilo (FICO), Gonçalo Mesquitela - e neri - Joana Simião, Uria Simango, Kawandame, Gumane - si riunirono in un'unica piattaforma politica per impedire l'applicazione dell'Accordo, il Movimento Mozambico Libero (Movimento Moçambique Livre, MML). A questo si associò un'organizzazione paramilitare denominata "Dragoni della Morte" (Dragões da Morte). Il Movimento Mozambico Libero scatenò, proprio nel giorno della firma dell'Accordo, una ribellione a Lourenço Marques, occupando Radio Club del Mozambico e, momentaneamente, l'aeroporto. L'MML riuscì anche a liberare circa duecento ex-agenti della polizia politica, che erano stati arrestati dopo il 25 aprile, e a mobilitare centinaia di manifestanti che sfilarono nelle vie della capitale mozambicana a favore del movimento. Attraverso Radio Club, ribattezzata Radio Libertà (Rádio Liberdade), i ribelli chiesero l'intervento sudafricano, le cui truppe erano posizionate sulla frontiera, a pochi chilometri da Lourenço Marques. Centinaia di manifestanti, soprattutto bianchi, protessero le stazioni dell'emittente da qualsiasi azione offensiva, mentre le entrate della città vennero sorvegliate da elementi della ex-OPVDC. A Beira e a Vila Pery si ebbero manifestazioni di appoggio agli insorti, $\mathrm{i}$ quali furono dispersi, anche un po' violentemente, dalle truppe portoghesi. Jorge Jardim, all'epoca in esilio in Malawi, era considerato il leader dei ribelli e fu varie volte indicato come il coordinatore esterno del movimento. La polizia e le forze militari portoghesi di stanza nella capitale mozambicana rimasero "indifferenti" di fronte agli avvenimenti,

\footnotetext{
Il 23 agosto 1974, la COREMO ed un insieme di piccoli partiti neri (FRECOMO, FUMO, MONIPAMO e MONAUMO) annunciarono la costituzione di una coalizione denominata Partito di Coalizione Nazionale (PCN). Furono scelti come Presidente Uria Simango e come Vice-Presidente Paulo José Gumane. Cfr. "Notícias», n. 16.235, 24 agosto 1974, 2.
} 
astenendosi dall'intervenire a favore o contro i ribelli. Anche Spínola si mostrò inizialmente riluttante ad agire contro gli insorti ${ }^{53}$.

Tuttavia, la situazione politica si alterò rapidamente a sfavore dei ribelli. Il 9 settembre 1974, Spínola, sotto pressione dell'MFA, ratificò l'Accordo di Lusaka, il che tolse agli insorti ogni speranza di appoggio da parte del Presidente della Repubblica. Anche i sudafricani optarono per non intervenire militarmente in Mozambico, offrendo solo asilo politico ai rivoltosi. Senza l'appoggio di vere forze militari e disponendo appena di un pugno di uomini armati, la ribellione era condannata a fallire. Così, l'11 settembre 1974, per ordine diretto del Generale Costa Gomes, truppe paracadutiste portoghesi, provenienti dal nord del Mozambico, misero la parola fine alla ribellione di Lourenço Marques. I dirigenti bianchi della rivolta riuscirono a fuggire verso il Sudafrica, mentre i leader neri si dettero alla clandestinità all'interno del paese e furono poi catturati dal Frelimo. La ribellione provocò un numero considerevole di morti e di feriti di tutte le razze. Provocò anche la reazione violenta di certi settori della popolazione nera, che si protrasse nelle settimane successive attraverso aggressioni fisiche, ruberie e distruzione di beni e proprietà dei bianchi. Quest'ondata di violenza razziale portò all'uscita "forzata" di migliaia di bianchi verso il Sudafrica e verso la Rhodesia [Saavedra 1975; Camilo 1984; Mesquitela 2014].

\section{Il Governo di Transizione e l'esodo dei bianchi}

L'Alto Commissario Vitor Crespo arrivò a Lourenço Marques il 12 settembre 1974, quattro giorni prima di Joaquim Chissano, che fu nominato Primo Ministro del Governo di Transizione il 21 settembre 1974.

53 PRO, FCO 9/2503, Overseas Territories of Portugal, 1974. Secondo il suo stesso racconto, Jorge Jardim era di passaggio a Johannesburg il giorno 7 settembre 1974. Cfr. Jardim 1976, 347-354. 
Dopo il primo mese di governo circa dodicimila militari portoghesi si erano ritirati dalle zone dell'interno. Alla fine di novembre del 1974, cinque delle otto province mozambicane erano governate da africani indicati dal Frelimo. Fu parimenti creata una Banca Centrale in sostituzione del Banco Nacional Ultramarino (Banca Nazionale Ultramarina) come entità emittente e furono definite alcune responsabilità finanziarie del nuovo paese e della (ancora) potenza coloniale. L'economia mozambicana era in caduta libera, aggravata dall'uscita delle risorse umane più qualificate, specialmente bianche, che scappavano dalla violenza razziale.

Infatti, il 21 ottobre 1974, a seguito di una rissa tra militari, si verificò una nuova ondata di violenza, di saccheggi e di omicidi che colpì soprattutto la popolazione bianca. Cinquanta bianchi furono assassinati e molti di più aggrediti da gruppi di «banditi» prima che le forze militari ristabilissero l'ordine a Lourenço Marques. Di conseguenza, altre migliaia di rifugiati bianchi attraversarono le frontiere con il Sudafrica e la Rhodesia. Questi avvenimenti dettero anche un impulso all'esodo verso il Portogallo di bianchi, indiani, meticci e finanche di alcuni neri che non si identificavano con il Frelimo [Camilo 1984, 341-343; MacQueen 1998, 186-187].

In verità, la paura fu una delle principali cause dell'esodo della popolazione bianca. Per anni, la propaganda del regime salazarista aveva diffuso paura tra la popolazione bianca, descrivendo i guerriglieri come un gruppo di terroristi. La continuazione della lotta armata da parte del Frelimo, dopo il 25 aprile, aveva avuto un impatto negativo sul comportamento della minoranza bianca, accentuandone i timori. Il tutto era stato aggravato dall'ambiente di scontro politico e sociale nelle principali città, soprattutto a partire dal giugno del 1974, così come dall'ondata di violenza che aveva interessato molti luoghi del territorio, soprattutto nel nord, nella regione di Nampula, dove erano avvenuti attacchi a popolazioni e famiglie bianche indifese da parte di gruppi armati sbandati nell'agosto del 1974. La ribellione di Lourenço Marques, 
il 7 settembre 1974, così come i disordini razziali che si susseguirono nei mesi seguenti, specificatamente in ottobre, furono la molla che fece scattare l'esodo dei coloni.

Ma si ebbero anche altri motivi che contribuirono all'uscita della popolazione bianca. Il modo in cui era stato portato a compimento il processo di decolonizzazione, per mezzo di un accordo stretto tra il Portogallo e il Frelimo, senza la partecipazione della società civile, aveva aumentato la sfiducia e l'incertezza nel futuro della maggioranza dei bianchi, che riteneva di non avere un posto nel paese dopo l'indipendenza. L'emarginazione politica dei partiti formati dai coloni, l'esilio di Jorge Jardim (uno dei più carismatici leader politici bianchi), il trasferimento del potere in regime di monopolio al Frelimo, tutto questo aveva portato all'esodo progressivo della minoranza bianca. Si aggiunga anche il fatto che l'adozione di politiche economiche di matrice marxista-leninista da parte del governo del Frelimo aveva progressivamente allontanato molti bianchi inizialmente rimasti nel paese, poi partiti poco dopo l'indipendenza.

Infatti, nel luglio del 1975, il Frelimo procedette alla nazionalizzazione dei servizi sociali e del sistema educativo del paese, il che generò risentimento tra molti bianchi, che non accettavano che i loro figli ricevessero un'educazione comunista. Mesi dopo, nel febbraio del 1976, terre ed edifici furono nazionalizzati, il che mise ancora più in fuga i bianchi. Nel gennaio del 1978, anche le banche portoghesi con attività nel paese furono nazionalizzate, il che provocò la reazione del governo di Lisbona. In quel momento, circa duecento cittadini portoghesi si trovavano incarcerati nelle prigioni mozambicane senza giudizio e ci furono esecuzioni con l'accusa di attività ribelli ${ }^{54}$. L'esodo dei bianchi fu dunque il risultato di un insieme di fattori e di opzioni politiche che non tennero nella giusta considerazione le specificità della minoranza bianca, che

54 Sulla questione della violenza politica in Mozambico si veda Macamo 2016, 85105. 
per la sua condizione di popolazione colonizzatrice, o sua discendente, non si poneva sullo stesso piano politico della maggioranza colonizzata [Rita-Ferreira 1988, 121-169].

Il 25 giugno 1975, il Mozambico abbracciò la sua indipenden$z a$ in una cerimonia presenziata dal Primo Ministro del Portogallo, Vasco Gonçalves. Samora Machel, che era arrivato due giorni prima nella capitale mozambicana, dopo un "pomposo" viaggio attraverso il territorio, diventò il primo Presi-

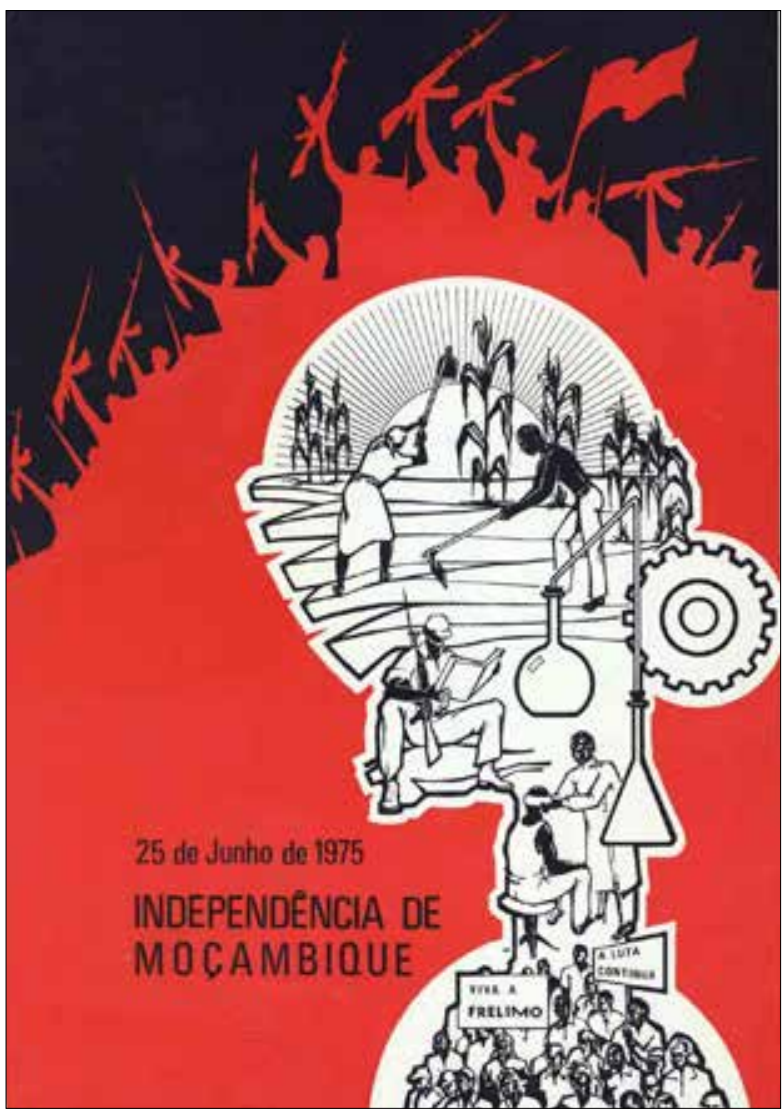

Direcçao Nacional de Propaganda e Publicidade, Giorno dell'indipendenza del Mozambico, 25 Gingno 1975. Grafica José Freire. Mozambico, 1975. Fonte: International Institute of Social History, call nr: BG E9/737 dente della Repubblica

Popolare del Mozambico. Tre anni dopo, il paese precipitò in una sanguinosa guerra civile che oppose il regime del partito unico del Frelimo ai ribelli della Resistenza Nazionale Mozambicana (Resistência Nacional Moçambicana, RENAMO), questi ultimi appoggiati dalla Rhodesia e dall'Africa del Sud. La pace e l'istituzione di un regime multipartitico sopraggiungeranno solo negli Anni ‘90, con l’Accordo di Roma (1992) e la realizzazione delle prime elezioni libere (1994). 


\section{Fonti primarie}

"Jornal Português de Economia \& Finanças" [1974]

«Notícias» [1974]

Public Record Office (PRO)

PRO, FCO 9/2049, Overseas Territories of Portugal, 1974.

PRO, FCO 9/2065, Relations Between Portugal and Africa, 1974 (British High Commission, Blantyre, 31/07/1974).

PRO, FCO 9/2503, Overseas Territories of Portugal, 1974

PRO, FCO 45/1533, Policy of Portugal to Her Overseas Territories, 1974.

\section{Bibliografia}

Afonso A., Gomes C. M. 2010, Os Anos da Guerra Colonial, 1961-1974, Porto: Quidnovi.

Aguiar, L. 1977, Livro Negro da Descolonização, Lisboa: Intervenção.

Antunes J. F. 1996, Jorge Jardim: agente secreto, Lisboa: Bertrand.

Bragança A., Wallerstein I. 1982, The African Liberation Reader, Londres: Zed.

Camilo C. 1984, Moçambique. Os acontecimentos de 7 de Setembro e 21 de Outubro de 1974, in Associação 25 de Abril (ed.) 1984, Seminário 25 de Abril. 10 anos depois, Lisboa: Fundação Calouste Gulbenkian, 341-344.

Carvalho O. S. 1991, Alvorada em Abril, Lisboa: Alfa.

Castelo C. 2007, Passagens para África. O Povoamento de Angola e de Moçambique com naturais da metrópole, Porto: Afrontamento.

Castilho J. M. T. 2012, Marcello Caetano - uma biografia política, Coimbra: Almedina.

Cruz M. B. 1980, Origens da democracia cristã e o salazarismo, Lisboa: Presença.

Dias J. 1964, Os Macondes de Moçambique, Lisboa: FLUL.

Hedges D., Universidade Eduardo Mondlane 1993, História de Moçambique. Moçambique no auge do colonialismo, 1930-1961, Maputo: Universidade Eduardo Mondlane.

Hastings A. 1974, Wiriyamu, Londres: Search Press.

Isaacman A., Isaacman B. 1983, Mozambique. From Colonialism to Revolution, 19001982, Boulder/Colorado: Westviem Press.

Jardim J. 1976, Moçambique terra queimada, Lisboa: Editorial Intervenção, 1976. 
Lloyd-Jones, S., Pinto, A. C. 2003, The Last Empire: Thirty Years of Portuguese Decolonization, Bristol: Intellect.

Macamo E. 2016, Violence and political culture in Mozambique, «Social Dynamics», 42 (1): $85-105$.

MacQueen N. 1998, A Descolonização da África Portuguesa, Mem Martins: Inquérito.

Mesquitela C. 2014, Moçambique - 7 de Setembro: Memórias da Revolução, Lisboa: Branco Editores.

Moita L. 1984, Não há uma, mas várias descolonizações, in Associação 25 de Abril (ed.) 1984, Seminário 25 de Abril. 10 anos depois, Lisboa: Fundação Calouste Gulbenkian, 287-290.

Mondlane E. 1969, The Stuggle for Mozambique, Harmondsworth: Penguin.

Morier-Genoud E. 2012, Sure Road? Nationalisms in Angola, Guinea-Bissau and Mozambique, Leiden\&Boston: Brill.

Newitt M. 1995, A History of Mozambique, London: C. Hurst \& Co.

Opello W. C. 1975, Pluralism and Elite Conflict in an Independence Movement: Frelimo in the 1960s, «Journal of Southern African Studies», 2 (1): 62-82.

Passerini L. 1970, Colonialismo Portoghese e Lotta di Liberazione nel Mozambico, Torino: Einaudi.

Pimenta F. T. 2010, Portugal e o Século XX. Estado-Império e Descolonização, 18901975, Porto: Afrontamento.

- 2011, Storia Politica del Portogallo Contemporaneo (1800-2000), Firenze: Le Monnier.

- 2012, Os Nacionalismos no Império: Angola e Moçambique in Jerónimo M. B. (ed.) 2012, O Império Colonial em Questão, Lisboa: Edições 70, 65-91.

- 2014, Emigração madeirense para Angola e Moçambique (1930-1948). O Caso do Arquivo da Agência Ferraz, «Islenha», 54: 93-110.

- 2016, Decolonisation Postponed: the Failure of the Colonial Politics of Marcelo Caetano, «Social Dynamics》, 42 (1): 12-30.

Pinto A. C. 1992, O salazarismo e o fascismo europeu, Lisboa: Estampa.

Rita-Ferreira A. 1988, Moçambique post-25 de Abril. Causas do êxodo da população de origem europeia e asiática, in Semana de Cultura Africana, Universidade de Coimbra (ed.) 1988, Moçambique: cultura e história de um país, Coimbra: Instituto de Antropologia, Universidade de Coimbra, 121-169.

Rodrigues L. N. 2010, Spinola, Lisboa: A Esfera dos Livros.

Rosas F. 1990, Portugal e o Estado Novo, 1930-1960, Lisboa: Presença.

Ruas H. B. (s/d), A Revolução das Flores. O Governo de Vasco Gonçalves até ao Acordo de Lusaka, Lisboa: Aster. 
Saavedra R. 1975, Aqui Moçambique Livre, Johannesburg: Livraria Moderna.

Santos A. A. 1975, 15 meses no governo ao serviço da descolonização, Lisboa: Asa.

- 2006, Quase memórias. Vol. 1: Do colonialismo e da descolonização. Vol. 2: Da descolonização de cada território em particular, Lisboa: Casa das Letras/Notícias.

- 2016, Gritante Justiça. Textos proibidos do meu tempo de Moçambique, Lisboa: Dom Quixote.

Souto A. N. 2007, Caetano e o ocaso do Império. Administração e guerra colonial em Moçambique durante o Marcelismo (1968-1974), Porto: Afrontamento.

Spínola A. 1974, Portugal e ofuturo, Lisboa: Arcádia.

Spínola A. 1978, País sem rumo: contributo para a história de uma revolução, Lisboa: Scire.

Torgal L. R. 2009, Estados Novos, Estado Novo, vols. 2, Coimbra: Imprensa da Universidade de Coimbra. 